\title{
Detection-confirmation-standardization-quantification: a novel method for the quantitative analysis of active components in traditional Chinese medicines
}

\author{
Xiaohong Song ${ }^{\text {Equal first author, } 1}{ }^{1}$, Anyi Zhao ${ }^{\text {Equal first author, } 1}{ }^{1}$, Yan Liu ${ }^{1}$, Jintang Cheng ${ }^{1}$, Sha Chen ${ }^{\text {Corresp., } 1}$, An Liu ${ }^{\text {Corresp. } 1}$ \\ ${ }^{1}$ Key Laboratory of Beijing for Identification and Safety Evaluation of Chinese Medicine, Institute of Chinese Materia Medica, China Academy of Chinese \\ Medical Sciences, Beijing, China \\ Corresponding Authors: Sha Chen, An Liu \\ Email address: schen@icmm.ac.cn, aliu@icmm.ac.cn
}

Background. Quantitative analysis of the active ingredients of Traditional Chinese Medicine is a research tendency. The objective of this study was to build a novel method, namely, Detection-confirmationstandardization-quantification (DCSQ) for the quantitative analysis of active components in traditional Chinese medicines, without individual reference standard.

Methods. Danshen (the dried root of Radix Salvia miltiorrhiza) was used as the matrix. The "extraction" function in high-performance liquid chromatography-mass (HPLC-MS) instrument was used to find the peaks corresponding to cryptotanshinone, tanshinone I, and tanshinone IIA in the total ion current (TIC) chromatogram of Danshen. The multicomponent reference standard (MCRS) containing the three tanshinones mainly was prepared by preparative HPLC. The contents of them in the resulting MCRS were determined by NMR; moreover, the constituents of the MCRS were confirmed. The MCRS containing known content of the three tanshinones was used as the reference standard for the quantitative analysis of cryptotanshinone, tanshinone I, and tanshinone IIA in Danshen Samples by analytical HPLC.

Results. Establishing the optimized HPLC conditions for the quantitative analysis of the active components in Danshen. And the assignments of the extracted peaks were confirmed by analyzing the characteristic fragments in their MS/MS product ion spectra and UV spectra. Then the MCRS containing the three tanshinones was prepared successfully. The results of determination about contents by NMR showed linearity fitted with high likelihood and calibration curves possessed high linearity. The results of determination on Danshen Samples obtained through DCSQ exhibited minimal deviations, in contrast to those obtained through individual reference standards.

Conclusion. The establishing DCSQ is independent and convenient for the quantitative analysis of the active components in TCMs by MCRS, without individual reference standard. This method is a great advance in quantitative analysis for complex composition, especially TCMs. 


\section{Detection-Confirmation-Standardization-}

\section{Quantification: A novel method for the quantitative}

3 analysis of active components in traditional Chinese

4 medicines

Xiaohong Song ${ }^{1 *}$, Anyi Zhao ${ }^{1 *}$, Yan Liu ${ }^{1}$, Jintang Cheng ${ }^{1}$, Sha Chen ${ }^{1}$ and An Liu ${ }^{1}$

${ }^{1}$ Key Laboratory of Beijing for Identification and Safety Evaluation of Chinese Medicine, Institute of Chinese Materia Medica, China Academy of Chinese Medical Sciences, Beijing, China

Background. Quantitative analysis of the active ingredients of Traditional Chinese Medicine is a research tendency. The objective of this study was to build a novel method, namely, Detectionconfirmation-standardization-quantification (DCSQ) for the quantitative analysis of active components in traditional Chinese medicines, without individual reference standard. Methods. Danshen (the dried root of Radix Salvia miltiorrhiza) was used as the matrix. The "extraction" function in high-performance liquid chromatography-mass (HPLC-MS/MS) instrument was used to find the peaks corresponding to cryptotanshinone, tanshinone I, and tanshinone IIA in the total ion current (TIC) chromatogram of Danshen. The multicomponent reference standard (MCRS) containing the three tanshinones mainly was prepared by preparative HPLC. The contents of them in the resulting MCRS were determined by NMR; moreover, the constituents of the MCRS were confirmed. The MCRS containing known content of the three tanshinones was used as the reference standard for the quantitative analysis of cryptotanshinone, tanshinone I, and tanshinone IIA in Danshen Samples by analytical HPLC.

Results. Establishing the optimized HPLC conditions for the quantitative analysis of the active components in Danshen. And the assignments of the extracted peaks were confirmed by analyzing the characteristic fragments in their MS/MS product ion spectra and UV spectra. Then the MCRS containing the three tanshinones was prepared successfully. The results of determination about contents by NMR showed linearity fitted with high likelihood and calibration curves possessed high linearity. The results of determination on Danshen Samples obtained through DCSQ exhibited minimal deviations, in contrast to those obtained through individual reference standards. 
Keywords. Quantitative analysis, Detection-confirmation-standardization-quantification (DCSQ), Multicomponent reference standard (MCRS), Tanshinones, HPLC-MS/MS, NMR

\section{Introduction}

Traditional Chinese medicines (TCMs) have been widely used in the treatment of various diseases because of their remarkable and reliable biological activities. Therefore, the determination of the active components in TCMs by chromatography methods such as highperformance liquid chromatography (HPLC), thin-layer chromatography (TLC), and highperformance capillary electrophoresis (HPCE) are considered as the main strategy by which the quality of TCMs may be controlled. Conventionally, the active components of TCMs are quantified by the corresponding reference standards(National Commission of Chinese Pharmacopoeia, 2020). Popular compounds are purchased from authoritative organizations, whereas rare compounds are purified inhouse. Another approach, namely, single standard to

- determine multicomponents (SSDMC), had been developed to reduce the reliance of quantity on the reference standards (Fang et al., 2017; Liu et al.,2017). The reference standards are still needed when assigning peaks and calculating conversion factors (the molar response ratio of reference standard to analyte).

NMR spectroscopy is considered as a promising quantification method for the direct determination of target compounds in mixtures without reference standards; the amount of analytes can be calculated using the ratios of the signal intensities of the protons of different compounds and internal reference standard(Frezza et al., 2018; Luisa et al., 2017; Petrakis et al., 2017; Chauthe et al., 2012; Staneva et al., 2011). However, a major challenge associated with NMR is the difficulty involved in the quantification of complex mixtures. The NMR spectra of complex mixtures often exhibit severe peak overlap, thereby affecting the accuracy of the quantity of analyte. To prevent severe peak overlap, Staneva et al.(2011) fractionated the extracts of TCMs before quantifying the target components by NMR(Chauthe et al., 2012; Staneva et al., 2011). This procedure often leads to the dissociation of target components into separate parts or absorption by sorbents. Consequently, the results do not accurately reflect the contents of target compounds in TCMs.

We proposed a novel method for the quantitative analysis of the active components in TCMs without individual reference standards, namely, detection-confirmation-standardizationquantification (DCSQ). Danshen (the dried root of Salvia miltiorrhiza), which was a popular traditional Chinese medicinal herb best known for its putative cardioprotective and antiatherosclerotic effects, was used as the matrix(Jia et al., 2019; Liu et al., 2015; Wang et al., 2011). The main components responsible for its pharmacological properties were hydrophilic depsides and lipophilic diterpenoid quinines(Wang et al., 2013; Li et al., 2018). An application of the DCSQ method for the quantitative analysis of three main diterpenoid quinones, cryptotanshinone, tanshinone I, and tanshinone IIA (Fig. 1, A-C) was reported for the first time. 
HPLC is a suitable technique for the quantitative analysis of the active components in TCMs because they contain numerous compounds. The separation of target components from a complex mixture in TCMs can be achieved by optimizing the HPLC conditions. Based on the combination of HPLC, MS, and NMR techniques, the quantitative analysis of the target components in TCMs without reference standard can be performed as follows: First, the "extraction" function in HPLC-MS instrument was used to find the peaks corresponding to the target components from the complex TIC chromatogram of TCMs, even without adequate separation( Wang et al.,2017). Next, the peaks were confirmed by the analysis of their MS/MS spectra. Finally, the HPLC conditions were optimized for the quantitative analysis of target components. The mixture mainly consisting of the target components, referred to as the multicomponent reference standard (MCRS), was prepared by preparative HPLC. The contents of the target components in the MCRS were determined directly by NMR, and the MCRS was used as the reference standard instead of individual reference standards.

\section{Materials \& Methods}

\section{Materials and Chemicals}

Danshen was purchased from Qiancao herbal wholesale company (Beijing). The standards of cryptotanshinone (1), tanshinone I (2), and Tanshinone IIA (3) (Fig. 1, A-C) were obtained from Traditional Chinese Medicine Solid Preparation National Engineering Research Center (Nangchang, Jiangxi Province, China), with a purity of $\geq 98 \%$. Dimethyl fumarate (4) (Fig. 1, D) with a purity of $99 \%$ was obtained from Alfa Aesar (Ward Hill, Massachusetts, USA). $\mathrm{CDCl}_{3}$ (99.8\% pure) was obtained from Cambridge Isotope Laboratories Inc. (Andover, MA, USA). Acetonitrile and methanol for HPLC were obtained from Fisher Scientific (Fair Lawn, New Jersey, USA). Formic acid ( $>98 \%$ purity) for HPLC was obtained from Sinopharm Chemical Reagent Co. Ltd. (Shanghai, China). All other chemicals were of analytical grade. Deionized water was obtained from Wahaha Company (Hangzhou, Zhejiang, China).

\section{Identification of cryptotanshinone, tanshinone I, and tanshinone IIA in Danshen by HPLC- ESI-MS/MS}

Sample preparation: Dried Danshen samples were powdered using a mill and sieved through a No. 24 mesh. Approximately $0.3 \mathrm{~g}$ of the powder sample was weighed and extracted by refluxing in $50 \mathrm{~mL}$ of methanol for $1 \mathrm{~h}$. The weight loss was adjusted with methanol after the extraction. One $\mathrm{mL}$ of the sample solution was filtered through a $0.45 \mu \mathrm{m}$ nylon filter into a HPLC amber sample vial for injection.

Data acquisition: An Agilent 1100 series HPLC system (Agilent Technologies, Santa Clara, CA, USA) equipped with a quaternary solvent delivery system, an on-line degasser, an autosample injector, a column temperature controller, and a variable wavelength detector (VWD) was coupled to an Agilent 6460 triple quadruple mass spectrometer (QQQ-MS) equipped with a dual electrospray ion source (ESI) (Agilent Technologies, Santa Clara, CA, USA) formed the HPLC-ESI-MS/MS system. 
115 The samples were separated using a Diamonsil C18 column $(4.6 \times 250 \mathrm{~mm}, 5 \mu \mathrm{m}$, Dikma 116 Technologies Inc.). The mobile phase was a mixture of acetonitrile (mobile phase A) and water 117 containing $0.1 \%$ formic acid (mobile phase $\mathrm{B}$ ). The gradient elution started at $60 \% \mathrm{~A}$, followed 118 by a linear increase to $80 \% \mathrm{~A}$ at $30 \mathrm{~min}, 90 \% \mathrm{~A}$ at $40 \mathrm{~min}$, a linear decrease to $60 \% \mathrm{~A}$ at $41 \mathrm{~min}$, 119 and held constant at 55 min before the next injection was performed. The flow rate was $1.0 \mathrm{~mL}$ $120 \mathrm{~min}^{-1}$. The column temperature was maintained at $22{ }^{\circ} \mathrm{C}$. The injection volume was $20 \mu \mathrm{L}$.

121 The mass spectrometric data were acquired using the positive electrospray mode. The full122 scan mass spectrum was recorded over the range of $m / z 250-325 . \mathrm{N}_{2}$ was used as the sheath and 123 auxiliary gas of the mass spectrometer. The capillary voltage for ESI spectra was $3.5 \mathrm{kV}$, and the 124 capillary temperature was set at $300^{\circ} \mathrm{C}$. Ultra-high pure helium was used as the collision gas in 125 the collision-induced dissociation (CID) experiments. The MS/MS product ion spectra were 126 acquired through the CID of the peaks with the protonated molecular ion $[\mathrm{M}+\mathrm{H}]^{+}$of each 127 analyte. The collision energy for the target protonated molecular ions was set at $20 \mathrm{eV}$ to obtain 128 129 the appropriate fragment information.

\section{Quantitative determination of each tanshinones in multicomponent reference standard by} QNMR

Generation of NMR calibration series: A series of volumetric solutions containing 20.50, 10.25, $6.83,3.42$, and $1.71 \mathrm{mg} / \mathrm{mL}$ of the MCRS and 2.96, 1.48, 0.74, 0.37 , and $0.19 \mathrm{mg} / \mathrm{mL}$ of dimethyl fumarate in $\mathrm{CDCl}_{3}$ were prepared and measured by NMR at $600 \mathrm{MHz}$.

NMR spectral acquisition and processing parameters: The spectra was acquired using a $14.1 \mathrm{~T}$ Bruker Avance $600 \mathrm{MHz}$ NMR spectrometer equipped with a $5 \mathrm{~mm}$ broad band (BB) inverse 
155

detection probe tuned to detect ${ }^{1} \mathrm{H}$ resonances. The ${ }^{1} \mathrm{H}$ resonance frequency was $600.13 \mathrm{MHz}$. All the spectra were acquired at $298 \mathrm{~K}$. A total of 64 scans of $32 \mathrm{~K}$ data points were acquired with a spectral width of $9615.4 \mathrm{~Hz}(16 \mathrm{ppm})$. A pre-acquisition delay of $6.5 \mu \mathrm{s}$, with an acquisition time of $1.7 \mathrm{~s}$, recycle delay of $24 \mathrm{~s}$, and flip angle of $90^{\circ}$ were used. The chemical shift of all the peaks was referenced to the tetramethylsilane (TMS) resonance at $0 \mathrm{ppm}$. The spectra were Fourier transformed to afford a digital resolution in the frequency domain of 0.293 $\mathrm{Hz} /$ Point. The phase and baseline corrections of the spectra were carried out manually. Preliminary data processing was carried out using the Bruker software TOPSPIN 2.1.

The signals for the H-15 of cryptotanshinone (4.884 ppm, t, 2H), H-17 of tanshinone I (2.295 ppm, d, 3H), H-17 of tanshinone IIA $(2.259 \mathrm{ppm}, \mathrm{d}, 3 \mathrm{H})$, and olefinic $\mathrm{H}$ of dimethyl fumarate $(6.864 \mathrm{ppm}, \mathrm{s}, 2 \mathrm{H})$ were used to determine the contents of cryptotanshinone, tanshinone I, and tanshinone IIA in the MCRS.

The concentrations of the three tanshinones in the MCRS were calculated using the following general equation:

$$
\mathrm{Cx}=\frac{\mathrm{Ax} \times \mathrm{Wi} \times \mathrm{Ni}}{\mathrm{Ai} \times \mathrm{Mi} \times \mathrm{Nx} \times \mathrm{V}}
$$

where $C_{x}$ (in $\mathrm{mM}$ ) corresponds to the concentrations of the three individual tanshinones; $A_{x}$ and $\mathrm{A}_{\mathrm{i}}$ correspond to the peak areas of tanshinones and the internal standard. $\mathrm{W}_{\mathrm{i}}$ corresponds to the mass of the internal standard (in $\mathrm{mg}$ ); $\mathrm{N}_{\mathrm{i}}$ and $\mathrm{N}_{\mathrm{x}}$ correspond to the number of protons of the respective signals of the internal standard and tanshinones used for the quantitative analysis; $M_{i}$ corresponds to the molecular weight (in Da) of the internal standard, and V (in $\mathrm{L}$ ) corresponds to the volume of $\mathrm{CDCl}_{3}$.

The contents of the three tanshinones in the MCRS were calculated using the following general equation:

$$
\mathrm{Px}=\frac{\mathrm{Cx} \times \mathrm{Mx} \times \mathrm{V}}{\mathrm{Wm}} \times 100 \%
$$

where $\mathrm{P}_{\mathrm{x}}(\%)$ corresponds to the percentage of the three individual tanshinones in the MCRS; $\mathrm{M}_{\mathrm{x}}$ corresponds to the molecular weight (in Da) of the three tanshinones, and $\mathrm{W}_{\mathrm{m}}$ corresponds to the mass of the MCRS (in mg).

\section{Quantitative analysis of the three tanshinones in the Danshen sample by HPLC using the MCRS as the reference standard}

Sample preparation and HPLC conditions: The Danshen sample was prepared according to the procedure described according to the above steps.

Analytical HPLC was carried out using a Shimadzu LC-20AT series equipped with a quaternary solvent delivery system, an on-line degasser, and auto-sample injector, a column temperature controller, and an SPD-M20A diode-array detector (DAD) (Shimadzu corporation, Kyoto, Japan). Finally, the samples were separated using a Diamonsil C18 column $(4.6 \times 250$ $\mathrm{mm}, 5 \mu \mathrm{m}$, Dikma Technologies Inc.). Notably, the HPLC conditions are compatible to the HPLC-ESI-MS experiments. 
193

194

195

196

197

198

199

200

201

202

203

204

205

206

207

208

209

210

211

212

213

214

215

216

217

218

219

220

221

222

223

224

225

226

227

228

Construction of calibration curves: Appropriate amounts of the MCRS were weighed and dissolved into $100 \mathrm{~mL}$ of acetone. To construct the calibration curves, $2,4,8,12,16$, and $20 \mu \mathrm{L}$ of the solution were injected in triplicates. The calibration curves were constructed by plotting the peak areas versus the quantity of the three tanshinones in the MCRS.

\section{Results}

Detection of the peaks corresponding to cryptotanshinone, tanshinone I, and tanshinone IIA from the TIC and HPLC chromatograms of Danshen

The initial investigation focused on determining the target components from the TIC and HPLC chromatograms of Danshen and establishing the optimized HPLC conditions suitable for the quantitative analysis of the active components in Danshen. A rough gradient elution was first performed, after which the peaks corresponding to cryptotanshinone, tanshinone I, and tanshinone IIA were searched with their protonated molecular ion $[\mathrm{M}+\mathrm{H}]^{+}$from the total ion chromatogram of Danshen( Wang et al.,2017). The results (Figs. 2, A and C) indicated that the peak with the retention times of $6.4,6.2$, and $8.5 \mathrm{~min}$ corresponded to the $\mathrm{m} / \mathrm{z}$ of 297,277 , and 295 , respectively. Under these conditions, cryptotanshinone and tanshinone I were not adequately separated, even though the positions of cryptotanshinone and tanshinone I in the TIC and HPLC spectra of Danshen were successfully assigned. The result obtained by the optimization of gradient elution is shown in Fig. 2 (B and D); the peaks with the retention times of 17.2, 19.0, and $26.1 \mathrm{~min}$ corresponded to the $\mathrm{m} / \mathrm{z}$ of 297,277 , and 295 , respectively, in the TIC of LC-MS (Fig. 2, B). Moreover, the three tanshinones were adequately separated from other compounds. This condition was adequate for the subsequent quantitative analysis of the three tanshinones in Danshen.

\section{Confirmation of the peak assignments by ESI-MS/MS product ion and UV spectra}

The ESI-MS/MS product ion spectra were acquired through the CID of the peaks obtained with the protonated molecular ion $[\mathrm{M}+\mathrm{H}]^{+}$of the three tanshinones. The proposed MS fragmentation pathway of the compounds with the protonated molecular ions of $m / z 297,277$, and 295 were summarized in Fig. 3, consistenting with the MS fragmentation pathway of cryptotanshinone, tanshinone I, and tanshinone IIA as studied by Wang et al., 2017. The UV spectra shown in Fig. 4 were obtained directly from the HPLC experiments. The UV spectra of the three peaks matched with those of cryptotanshinone, tanshinone I, and tanshinone IIA, as reported in the current literature(Huang et al., 2018).

Preparation of the MCRS containing mainly cryptotanshinone, tanshinone I, and tanshinone IIA 
229

230

231

232

233

234

235

236

237

238

239

240

241

242

243

244

245

246

247

248

249

250

251

252

253

254

255

256

257

258

259

260

261

262

263

264

265

266

267

268

The MCRS containing mainly cryptotanshinone, tanshinone I, and tanshinone IIA was prepared by the preparative HPLC of the total tanshinone to perform a quantitative analysis of the three components, without individual reference standards. The chromatograms of the total tanshinone and MCRS were shown in Fig. 5 (picture A). Peaks 1, 2, and 3 were assigned to cryptotanshinone, tanshinone I, and tanshinone IIA, respectively, by collecting the main peaks in the preparative HPLC separately and then analyzing them by analytical HPLC.

\section{Determination of the contents of cryptotanshinone, tanshinone I, and tanshinone IIA in the} MCRS by NMR

The contents of the three tanshinones in the resulting MCRS were determined directly by ${ }^{1} \mathrm{H}$ NMR analyses. The ${ }^{1} \mathrm{H}$ NMR spectrum of the MCRS mixed with the internal standard, dimethyl fumarate, was shown in Fig. 6. The main signals were assigned to the three tanshinones according to the references, excluding the signals belonging to dimethyl fumarate(Mei et al., 2019; Zeng et al., 2017; Wu et al., 2015). The composition of the MCRS was confirmed again by analyzing the signals observed in the ${ }^{1} \mathrm{H}$ NMR spectrum. To perform an accurate quantitative analysis, the data processing technique was used which named the Lorentz deconvolution function in the Bruker software TOPSPIN 2.1. The linearity of signals were fitted by the Lorentz method. Next, the deconvolution was performed, and the peak areas were automatically generated. Regarding the quantitative analysis of the mixtures by NMR using the data processing, the accuracy was determined by the likelihood of lineshape fitting. The results showed that the contents of crytotanshinone, tanshinone I, and tanshinone IIA were at 1.635 $\mathrm{g} / 100 \mathrm{~g}, 0.718 \mathrm{~g} / 100 \mathrm{~g}, 1.953 \mathrm{~g} / 100 \mathrm{~g}$ in Danshen sample, respectively, by MCRS method.

Under the preparation of MCRS of Danshen, the selection of the signals in the NMR spectra for the quantitative analysis of the three tanshinones and the validity of the NMR method were performed. The analytical method of NMR was achieved a high separation efficiency which was shown in Fig. 7 (A-O). The NMR spectrum is very reproducible with good reproducibility and target three compounds high separation efficiency.

Determination of the contents of the three tanshinones in the Danshen sample by HPLC using the MCRS as the reference standard and comparison of the results with those obtained using individual reference standards.

The calibration curve of the MCRS was constructed to investigate the validity of the NMR method. The calibration curves were constructed by plotting the concentrations of the three tanshinones versus that of dimethyl fumarate. Next, the linear regression lines were calculated. The calibration graph demonstrating the linearity of the NMR response with increasing concentrations of the three tanshinones was shown in Fig. 8.

Approximately $5 \mathrm{mg}$ of the MCRS was dissolved into $100 \mathrm{~mL}$ of acetone to construct the calibration curve of the three tanshinones, corresponding to $\sim 1.5,0.5$, and $1.7 \mathrm{mg}$ of individual cryptotanshinone, tanshinone I, and tanshinone IIA, respectively. The contents of the three tanshinones in the Danshen sample were determined according to the calibration curves based on 
269

270

271

272

273

274

275

276

277

278

279

280

281

282

283

284

285

286

287

288

289

290

291

292

293

294

295

296

297

298

299

300

301

302

303

304

305

306

307

individual standards. The content of cryptotanshinone was determined at $1.635 \mathrm{~g} / 100 \mathrm{~g}$ and 1.627 $\mathrm{g} / 100 \mathrm{~g}$, respectively, using MCRS and standard quantitation. Tanshinone I was detected at 0.718 $\mathrm{g} / 100 \mathrm{~g}$ and $0.727 \mathrm{~g} / 100 \mathrm{~g}$, respectively, based on the two methods. Tanshinone IIA was measured at $1.953 \mathrm{~g} / 100 \mathrm{~g}$ and $1.886 \mathrm{~g} / 100 \mathrm{~g}$, respectively. The mean contents of the three compounds obtained by the two methods were tested for no significance difference at the $P \leq 0.05$ level. The results were compared with those obtained using individual reference standards. The deviations of the three tanshinones determined by the two methods were minimal (Fig. 9), and this could be attributed to the maximum likelihood lineshape fitting of their signals in the NMR spectrum of the MCRS.

\section{Discussion}

The multicomponent reference standard (MCRS) of Danshen was preparing successfully to use for the samples' quantitative analysis. The MCRS containing cryptotanshinone, tanshinone I, and tanshinone IIA was obtained by collecting peaks 1, 2, and 3 together (Fig. 5, picture B). The preparation of MCRS was found to be much easier than that of individual reference standards. The presence of some other compounds whose NMR signals did not overlap severely with those of the target components may be allowed. And the target components need not be separated from each other in their preparative HPLC spectrum because they were finally collected for preparing the MCRS.

Apart from structure elucidation, NMR had been progressed to become a useful technique for the direct quantitative analysis of complex mixtures. However, it remained difficult to directly quantitatively analyze the target components from the extracts of TCMs using the NMR technique, owing to the existence of numerous components, which causing severe overlap of the NMR signals. In this study, HPLC was selected for the quantification of the three tanshinones in Danshen for its outstanding separation ability compared to NMR. By using the "extraction" function in HPLC-MS instrument to find the target components and MCRS as the reference standard diminished the dependency of individual reference standards in the quantitative analysis of the target components in TCMs. Evidently, the increased amount of components in TCM samples would not make the quantitative analysis of target components more difficult, which could be attributed to the efficient separation function of HPLC. The components whose signals overlap with each other could be divided into different MCRSs. The amount of MCRS prepared from one sample could be adjusted according to the components requiring quantification and considering the prevalence of NMR signal overlapping.

The results of MCRS shown in Fig. 7 indicated that the extents of the likelihood of the lineshape fitting of the signals for the three tanshinones performed by the Lorentz deconvolution are different. Pictures A-E, F-K, L-M, and N-O showed the lineshape fitting results of the signals for cryptotanshinone, tanshinone I, tanshinone IIA, and dimethyl fumarate, respectively. The lineshapes of the following signals were fitted with relatively higher likelihood: the triplet in $\mathbf{B}$ at $4.884 \mathrm{ppm}$ belonged to cryptotanshinone, the doublets in $\mathbf{G}$ and $\mathbf{K}$ (right side) belonged to 
308 tanshinone I, the doublet in K (left side) belongs to tanshinone IIA, and the singlet in $\mathbf{N}$ 309 belonged to dimethyl fumarate.

310 The calibration graph demonstrating the linearity of the NMR response with increasing 311 concentrations of the three tanshinones in Fig. 8 indicated that the $R^{2}$ values calculated from the 312 signals in $\mathbf{C}$ and $\mathbf{K}$ (left side) (Fig. 7) belonging to cryptotanshinone and tanshinone IIA were 3131.0000 and 0.9999, respectively. The signals in $\mathbf{G}$ and $\mathbf{K}$ (right side) (Fig. 7)belonging to 314 tanshinone I also exhibited a high likelihood of lineshape fitting, and the $R^{2}$ values calculated 315 from the two signals were 0.9995 and 0.9997 , respectively. The result confirmed that the 316 concentrations of the three tanshinones in the MCRS could be accurately determined using ${ }^{1} \mathrm{H}$

317 NMR after deconvolution. The average contents of three tanshinones was calculated with five 318 different concentrations from the NMR spectra of the MCRS. And the results were 28.01\%, $3199.43 \%$, and $34.36 \%$, respectively. The signals in B, K (left side and right side) (Fig. 7), and $\mathbf{N}$ 320 (Fig. 7) were finally selected for the quantitative analysis of the three tanshinones, because their 321 linearity fitted with high likelihood and their calibration curves possessed high linearity.

322 The calibration curves of the three tanshinones were constructed using the same method as 323 that of individual reference standards. A significant advantage of using the MCRS as the 324 reference standard was that it reduced the dependency of quantitative analysis on the individual 325 reference standard and also reduced the consumption of reference standards.

326

\section{Conclusions}

328

329

330

331

332 corresponding samples, which was less dependent than individual reference standards. The MCRS as the reference standard will be used to quantitative analysis and for the more industrial applications. It will be more accurate and convenient for the target analyte. It was a great advance in quantitative analysis for complex composition, especially TCMs. 
345 The authors are grateful for the financial support from "the Fundamental Research Funds for the

346 Central Public Welfare Research Institutes" (grant no. ZZ13-YQ-057) and National Science and

347 Technology Major Project for "Significant New Drugs Development" (grant no.

348 2019ZX09201005 and 2018ZX09735-

350 design, data collection and analysis,
353

354

355

356

357

358

359

360

361

362

363

364

365

366

367

368

369

370

371

372

373

374

375

376

377

378

379

380

381

382

383 manuscript.

\section{References}

1. National Commission of Chinese Pharmacopoeia. Pharmacopoeia of People's Republic of China, vol I. Beijing: China Medical Science Press; 2020: 77-79.

2. Fang J, Yang B, Ge Z, Bai X, Yan B. 2017. Single standard substance for the determination of nine volatile components in the distillate of Fructus Gardeniae and Radix Curcumae (an intermediate of Xingnaojing Injection). Journal of Separation Science 40:3946-3957. DOI 10.1002/jssc.201700593.

3. Liu W, Zhang JS, Han W, Liu YF, Feng J, Tang CH, Feng N, Tang QJ. 2017. One single standard substance for the simultaneous determination of 17 triterpenes in Ganoderma lingzhi and its related species using highperformance liquid chromatography. Journal of Chromatorgraphy B 1068:49-55. DOI 10.1016/j.jchromb.2017.10.010.

4. Frezza C, Venditti A, Sciubba F, Tomai P, Antonetti M, Franceschin M, Gentili A, Delfini M, Serafini M, Bianco A.2018. Phytochemical profile of Euphorbia peplus L. collected in Central Italy and NMR semiquantitative analysis of the diterpenoid fraction. Journal of Pharmaceutical and Biomedical Anlysis 160:152-159. DOI 10.1016/j.jpba.2018.07.059.

5. Luisa, Marina, Maria, Angeles, Garcia, Menendez-Lopez. 2017. Enantiomeric separation of the antiuremic drug colchicine by electrokinetic chromatography: Method development and quantitative analysis. Journal of Pharmaceutical and Biomedical Anlysis 138:189-196. DOI 10.1016/j.jpba.2017.02.001.

6. Petrakis EA, Cagliani LR, Tarantilis PA, Polissiou MG, Consonni R. 2017. Sudan dyes in adulterated saffron ( Crocus sativus L.): Identification and quantification by 1 H NMR. Food Chemistry 217:418-424. DOI 10.1016/j.foodchem.2016.08.078.

7. Chauthe SK, Sharma RJ, Aqil F, Gupta RC, Singh IP. 2012. Quantitative NMR: An Applicable Method for Quantitative Analysis of Medicinal Plant Extracts and Herbal Products. Phytochemical Analysis 23:689-696. DOI $10.1002 /$ pca. 2375 .

8. Staneva J, Denkova P, Todorova M, Evstatieva L. 2011. Quantitative analysis of sesquiterpene lactones in extract of Arnica montana L. by 1H NMR spectroscopy. Journal of Pharmaceutical and Biomedical Anlysis 54:94-99. DOI 10.1016/j.jpba.2010.08.018.

9. Jia D, Zhang CZ, Qiu Y, Chen XF, Jia L, Chen AF, Chai YF, Zhu ZY, Huang J, Zhang C. 2019. Cardioprotective mechanisms of salvianic acid A sodium in rats with myocardial infarction based on proteome and transcriptome analysis. Acta Pharmacologica Sinica 40:1513-1522. DOI 10.1038/s41401-019-0265-1.

10. Liu Z, Xu S, Huang X, Wang J, Liu P. 2015. Cryptotanshinone, an orally bioactive herbal compound from Danshen, attenuates atherosclerosis in Apolipoprotein E-Deficient Mice: role of LOX-1. British Journal of

Peer] An. Chem. reviewing PDF | (ACHEM-2020:11:55600:2:0:NEW 17 Feb 2021) 
384

385

386

387

388

389

390

391

392

393

394

395

396

397

398

399

400

401

402

403

404

405

406

407

408

409

410

411

412

413

414

415

416

417

418

419

420

421

422

423

424

425

426

427

428

429

Pharmacology 172:5661-5675. DOI 10.1111/bph.13068.

11. Wang XY, Wang Y, Jiang M, Zhu Y, Hu LM, Fan GW, Wang Y, Li XJ, Gao XM. 2011. Differential cardioprotective effects of salvianolic acid and tanshinone on acute myocardial infarction are mediated by unique signaling pathways. Journal of Ethnopharmacology 135:662-671. DOI 10.1016/j.jep.2011.03.070.

12. Wang Y, Peng H, Shen Y, Zhao R, Huang L. 2013. The profiling of bioactive ingredients of differently aged Salvia miltiorrhiza roots. Microscopy Reseach and Technique 76:947-954. DOI 10.1002/jemt.22253.

13. Li ZM, Xu SW, Liu PQ. 2018. Salvia miltiorrhiza Burge (Danshen): A golden herbal medicine in cardiovascular therapeutics. Acta Pharmacologica Sinica 39:802-824. DOI 10.1038/aps.2017.193.

14. Wang FR, Zhang Y, Yang XB, Liu CX, Yang XW, Xu W, Liu JX. 2017. Rapid Determination of 30 Polyphenols in Tongmai Formula, a Combination of Puerariae Lobatae Radix, Salviae Miltiorrhizae Radix et Rhizoma, and Chuanxiong Rhizoma, via Liquid Chromatography-Tandem Mass Spectrometry. Molecules 22:545. DOI 10.3390/molecules22040545.

15. National Commission of Chinese Pharmacopoeia. Pharmacopoeia of People's Republic of China, vol I. Beijing: China Medical Science Press; 2020: 416-417.

16. Huang H, Lv Y, Sun X, Fu S, Liu Z. 2018. Rapid determination of tannin in Danshen and Guanxinning injections using UV spectrophotometry for quality control. Journal of Innovative Optical Health Sciences 11:1850034. DOI 10.1142/S1793545818500347.

17. Mei XD, Cao YF, Che YY, Li J, Shang ZP, Zhao WJ, Qiao YJ, Zhang JY. 2019. Danshen: a phytochemical and pharmacological overview. Chinese Journal of Natural Medicines 17:59-80. DOI 10.1016/S18755364(19)30010-X.

18. Zeng HT, Su SL, Xiang X, Sha XX, Zhu ZH, Wang YY, Guo S, Yan H, Qian DW, Duan JN. 2017. Comparative Analysis of the Major Chemical Constituents in Salvia miltiorrhiza Roots, Stems, Leaves and Flowers during Different Growth Periods by UPLC-TQ-MS/MS and HPLC-ELSD Methods. Molecules 22:771. DOI 10.3390/molecules22050771.

19. Wu DF, Jiang XH, Wu SH. 2015. Direct purification of tanshinones from Salvia miltiorrhiza Bunge by high-speed counter-current chromatography without presaturation of the two-phase solvent mixture. Journal of Separation Science 33:67-73. DOI 10.1002/jssc.200900491.

2

(5)

7

8


430

431

432

433

434

435

436

437

438

439

440

441

442

443

444

445

446

447

448

449

450

451

452

453

454

455

456

457

458

459 460

461

462

463

464

465

466

467

468
Figure 1 Chemical structures of cryptotanshinone (A), tanshinone I (B), tanshinone IIA (C), and dimethyl fumarate (D).

Figure 2 Total ion current chromatograms of the Danshen extract and extracted ion chromatograms of target protonated molecular ions (A-B) and UV spectrum of the Danshen extract (C-D). The HPLC conditions were as follows: The water phase contained $0.1 \%$ formic water. The flow rate was $1.0 \mathrm{~mL} / \mathrm{min}$. The detection wavelength was set at $270 \mathrm{~nm}$. The eluting condition of (A-B) was as follows: $85 \%$ acetonitrile was maintained for $15 \mathrm{~min}$, and the column temperature was $35^{\circ} \mathrm{C}$. The eluting condition of (C-D) was as follows: $60 \%$ acetonitrile was maintained for $0-30 \mathrm{~min}$, and the concentration of acetonitrile was increased from $60 \%$ to $80 \%$ and then to $90 \%$ of acetonitrile at $40 \mathrm{~min}$. The column temperature was $22^{\circ} \mathrm{C}$.

Figure 3 Positive ion ESI-MS/MS spectra and the proposed MS fragmentation pathways of peaks 1 (A), 2 (B), and $3(\mathrm{C})$.

Figure 4 UV spectra of the three peaks shown in Fig. 2 (picture D). (A) UV spectrum of the peak with the retention time of $16.8 \mathrm{~min}$; (B) UV spectrum of the peak with the retention time of $18.6 \mathrm{~min}$; (C) UV spectrum of the peak with the retention time of $25.7 \mathrm{~min}$.

Figure 5 Representative preparative HPLC chromatogram of total tanshinones (A) and analytical HPLC chromatogram of the MCRS (B). The MCRS was analyzed under the same condition as HPLC-MS experiment (Fig. 2, eluting gradient of (C-D)).

Peer] An. Chem. reviewing PDF | (ACHEM-2020:11:55600:2:0:NEW 17 Feb 2021) 
470 Figure $6600 \mathrm{MHz}{ }^{1} \mathrm{H}$ NMR spectrum of the MCRS mixed with dimethyl fumarate. The concentrations 471 of the MCRS and dimethyl fumarate in $\mathrm{CDCl} 3$ were 20.50 and $2.96 \mathrm{mg} \cdot \mathrm{mL}^{-1}$, respectively.

472

473 Figure 7 Representative results of the lineshape fitting of the signals belonging to the three tanshinones 474 and dimethyl fumarate performed by the Lorentz deconvolution. Images A-E reflect the representative 475 lineshape fitting of signals belonging to cryptotanshinone. Images F-K (the right side) reflect the 476 representative lineshape fitting of signals belonging to tanshinone I. The doublet in the left side of K, 477 images $\mathrm{L}$ and $\mathrm{M}$ (the doublet in the right side) reflect the representative lineshape fitting of signals 478 belonging to tanshinone IIA. Images $\mathrm{N}$ and $\mathrm{O}$ reflect the lineshape fitting of signals belonging to dimethyl 479 fumarate.

480

481 Figure 8 Concentrations of the three tanshinones determined by specific signals.

482

483 Figure 9 Deviations of the contents of the three tanshinones in the Danshen sample as determined by the 484 MCRS contrast to individual reference standards. 


\section{Figure 1}

Figure 1 Chemical structures of cryptotanshinone (A), tanshinone I (B), tanshinone IIA (C), and dimethyl fumarate (D).

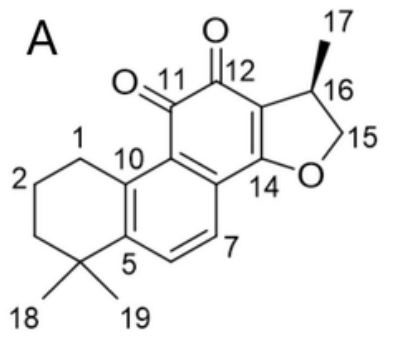

Cryptotanshinone (1) Chemical Formula: $\mathrm{C}_{19} \mathrm{H}_{20} \mathrm{O}_{3}$ Molecular Weight: 296.36
B<smiles>Cc1coc2c1C(=O)C(=O)c1c-2ccc2c(C)cccc12</smiles>

Tanshinone I (2) Chemical Formula: $\mathrm{C}_{18} \mathrm{H}_{12} \mathrm{O}_{3}$ Molecular Weight: 276.29<smiles>Cc1coc2c1C(=O)C(=O)c1c-2ccc2c1CCCC2(C)C</smiles>

Tanshinone IIA (3) Chemical Formula: $\mathrm{C}_{19} \mathrm{H}_{18} \mathrm{O}_{3}$ Molecular Weight: 294.34
D<smiles>COC(=O)C=CC(=O)OC</smiles>

Dimethyl fumarate (4) Chemical Formula: $\mathrm{C}_{6} \mathrm{H}_{8} \mathrm{O}_{4}$ Molecular Weight: 144.13 


\section{Figure 2}

Figure 2 Total ion current chromatograms of the Danshen extract and extracted ion chromatograms of target protonated molecular ions (A-B) and UV spectrum of the Danshen extract (C-D).

The HPLC conditions were as follows: The water phase contained $0.1 \%$ formic water. The flow rate was $1.0 \mathrm{~mL} / \mathrm{min}$. The detection wavelength was set at $270 \mathrm{~nm}$. The eluting condition of (A-B) was as follows: 85\% acetonitrile was maintained for $15 \mathrm{~min}$, and the column temperature was $35{ }^{\circ} \mathrm{C}$. The eluting condition of (C-D) was as follows: $60 \%$ acetonitrile was maintained for 0-30 min, and the concentration of acetonitrile was increased from $60 \%$ to $80 \%$ and then to $90 \%$ of acetonitrile at $40 \mathrm{~min}$. The column temperature was $22{ }^{\circ} \mathrm{C}$.
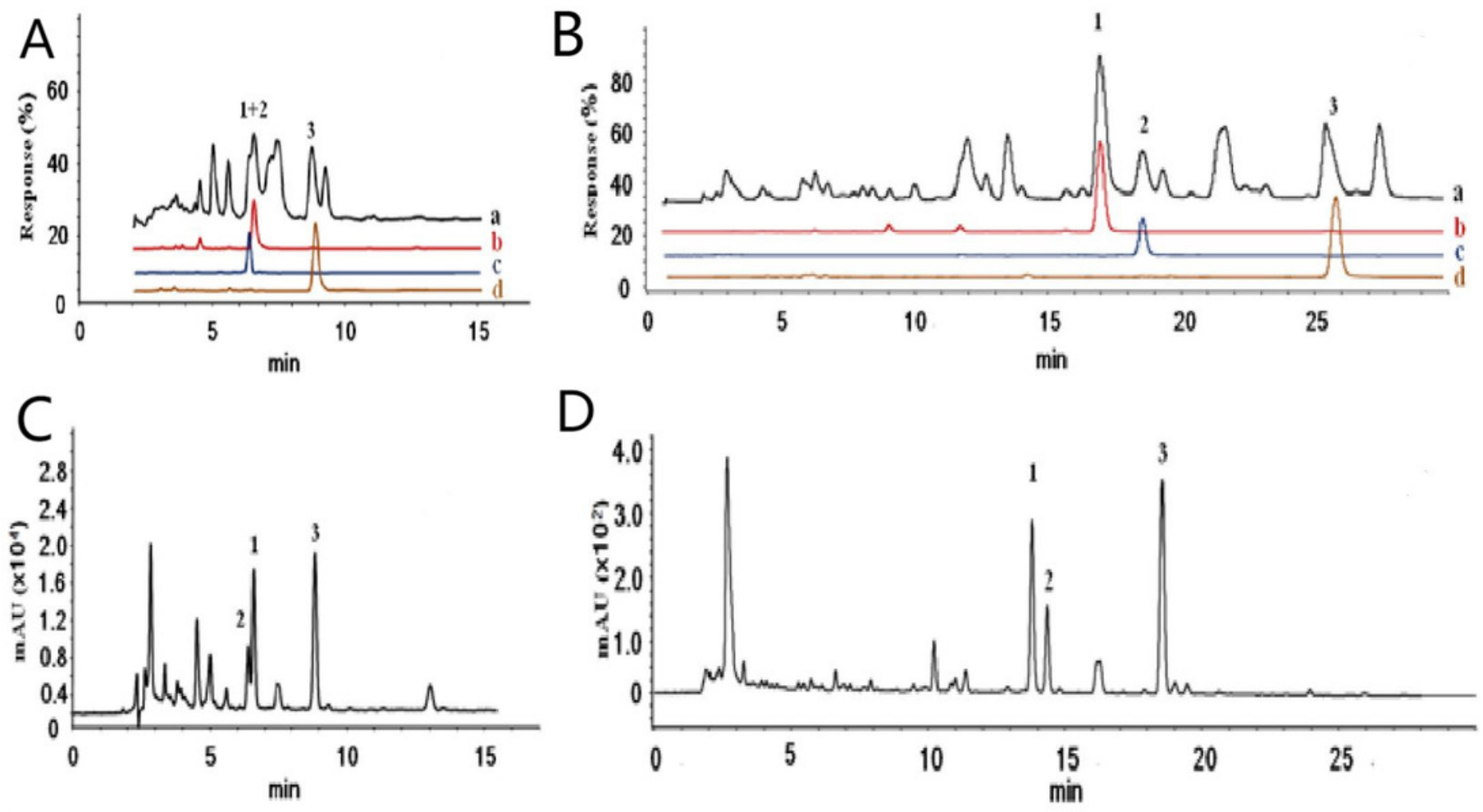
Figure 3

Figure 3 Positive ion ESI-MS/MS spectra and the proposed MS fragmentation pathways of peaks $1(A), 2(B)$, and $3(C)$.

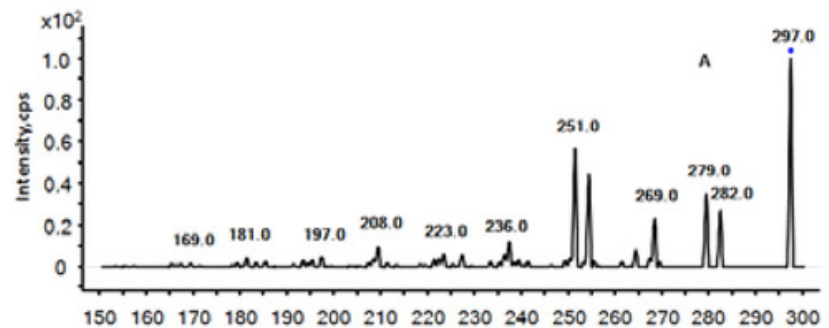

$\mathrm{m} / \mathrm{z}$
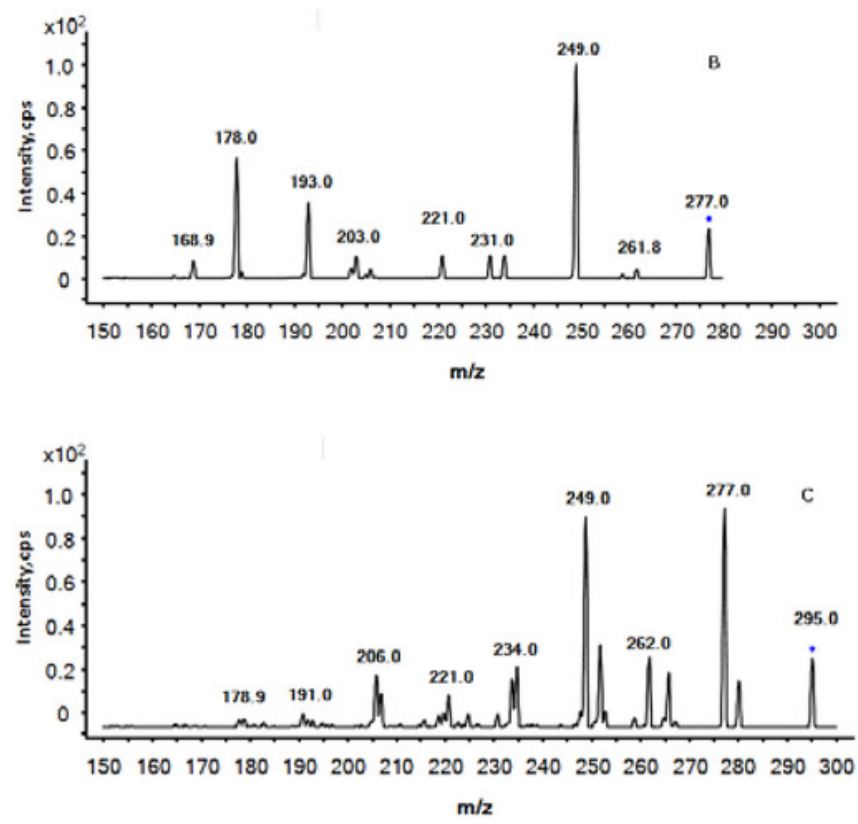

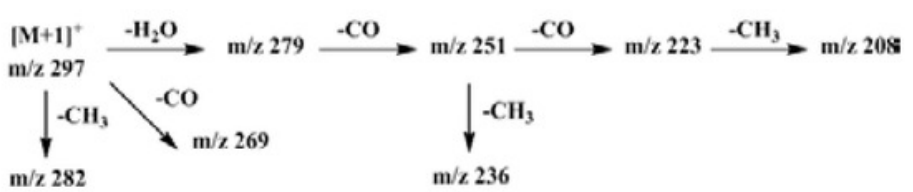

$\underset{\mathrm{m} / \mathrm{z} 277}{[\mathrm{M}+1]^{+}} \stackrel{-\mathrm{CO}}{\longrightarrow} \mathrm{m} / \mathrm{z} 249 \stackrel{-\mathrm{CO}}{\longrightarrow} \mathrm{m} / \mathrm{z} 221 \stackrel{-\mathrm{CO}}{\longrightarrow} \mathrm{m} / \mathrm{z} 193 \stackrel{\mathrm{CH}_{3}}{\longrightarrow}-\mathrm{m} / \mathrm{z} 178$
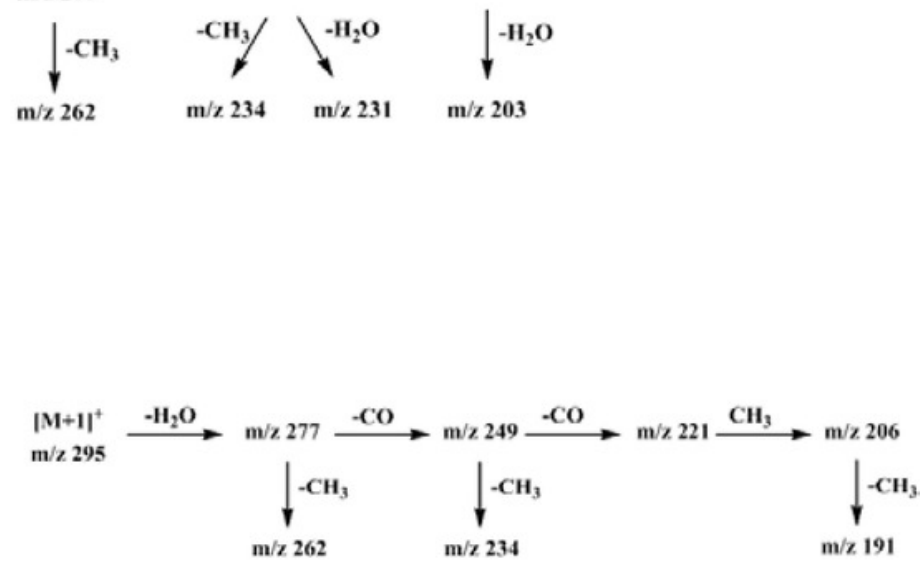
Figure 4

Figure 4 UV spectra of the three peaks

UV spectra of the three peaks shown in Fig. 2 (picture D). (A) UV spectrum of the peak with the retention time of $16.8 \mathrm{~min}$; (B) UV spectrum of the peak with the retention time of 18.6 min; (C) UV spectrum of the peak with the retention time of $25.7 \mathrm{~min}$.
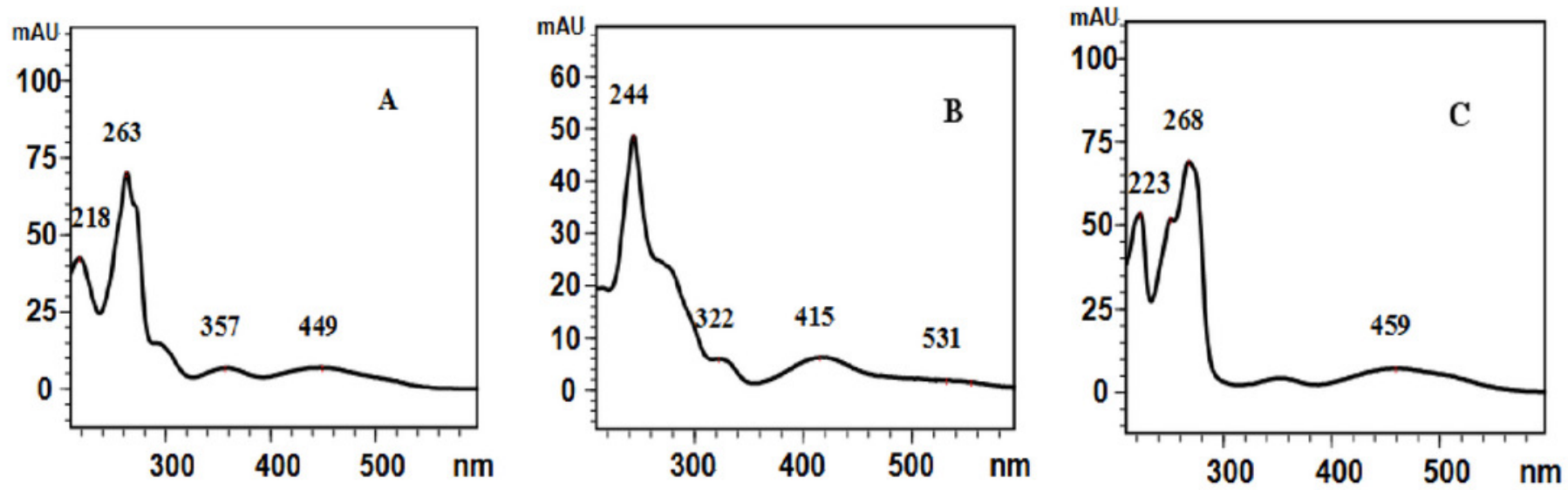


\section{Figure 5}

Figure 5 Representative preparative HPLC chromatogram of total tanshinones (A) and analytical HPLC chromatogram of the MCRS (B).

The MCRS was analyzed under the same condition as HPLC-MS experiment (Fig. 2, eluting gradient of (C-D) ). 

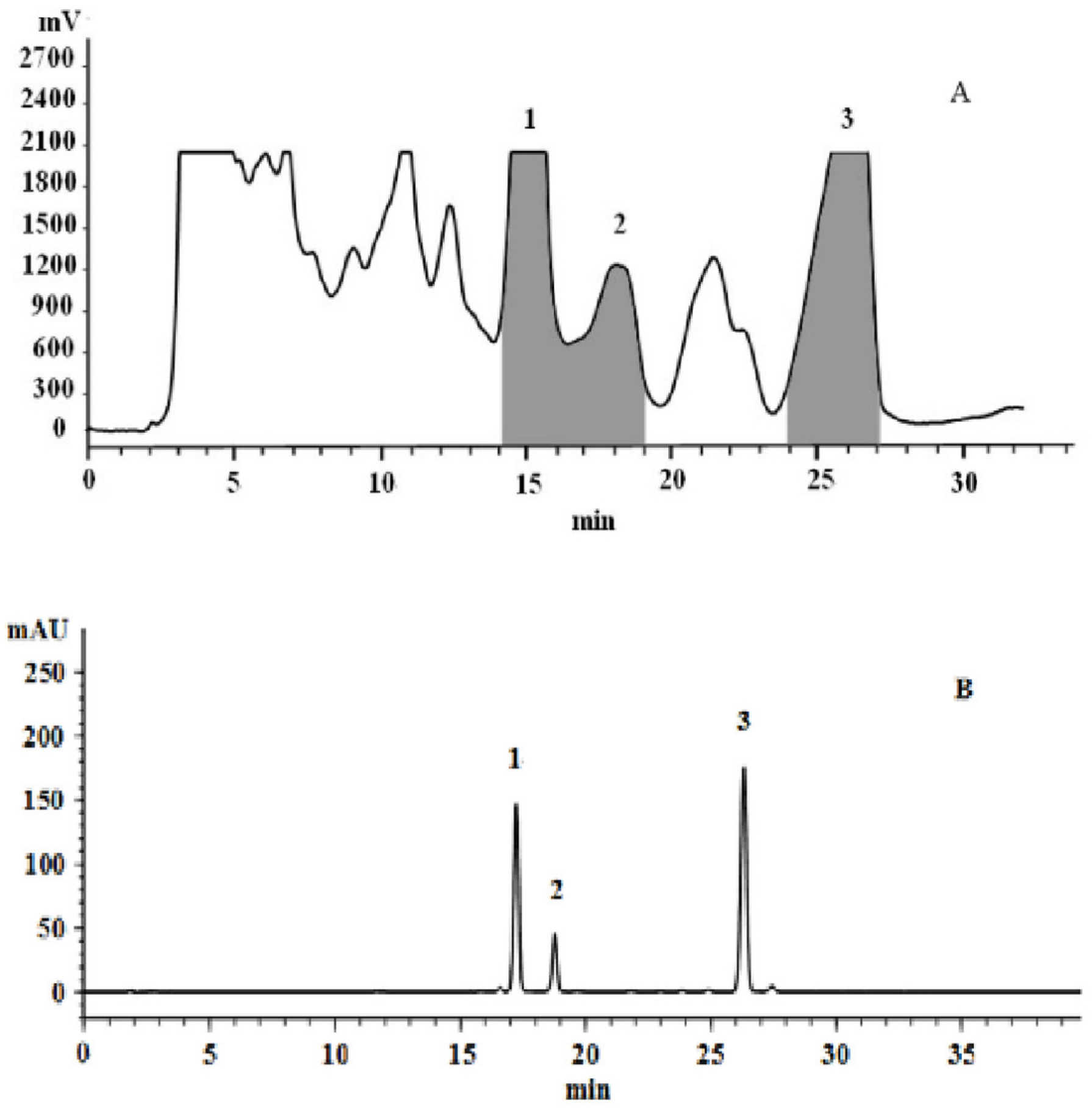
Figure 6

Figure $6600 \mathrm{MHz}{ }^{1} \mathrm{H}$ NMR spectrum of the MCRS mixed with dimethyl fumarate.

The concentrations of the MCRS and dimethyl fumarate in $\mathrm{CDCl} 3$ were 20.50 and 2.96 $\mathrm{mg} \cdot \mathrm{mL}^{-1}$, respectively.






\section{Figure 7}

Figure 7 Representative results of the lineshape fitting of the signals belonging to the three tanshinones and dimethyl fumarate performed by the Lorentz deconvolution.

Images A-E reflect the representative lineshape fitting of signals belonging to cryptotanshinone. Images F-K (the right side) reflect the representative lineshape fitting of signals belonging to tanshinone $I$. The doublet in the left side of $K$, images $L$ and $M$ (the doublet in the right side) reflect the representative lineshape fitting of signals belonging to tanshinone IIA. Images $\mathrm{N}$ and $\mathrm{O}$ reflect the lineshape fitting of signals belonging to dimethyl fumarate.

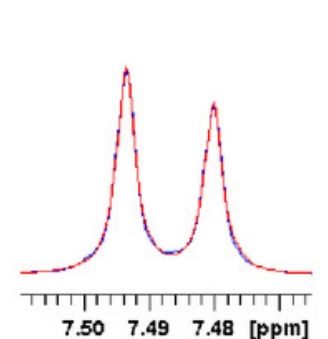

A
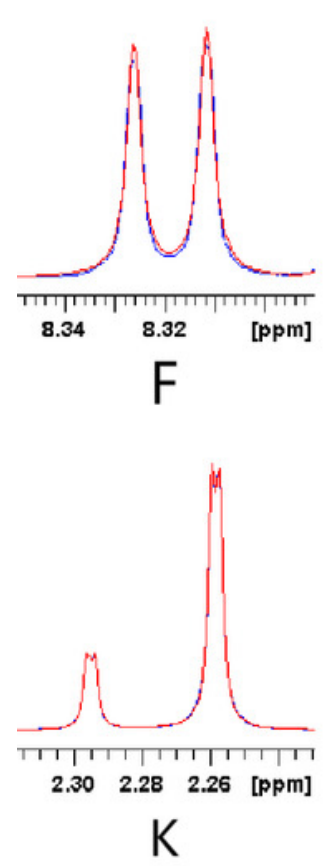

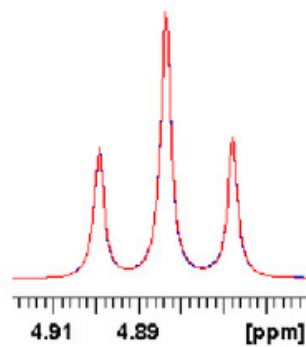

B
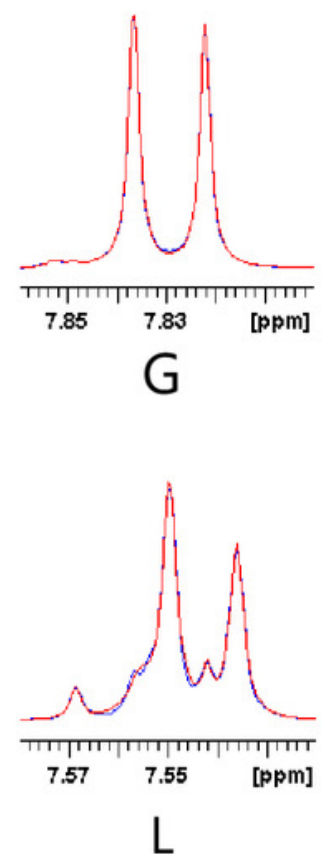
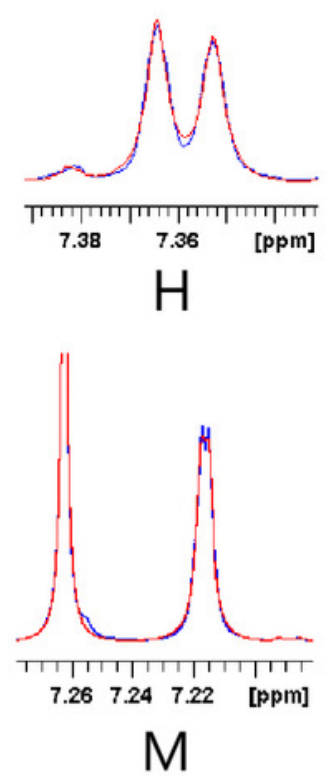
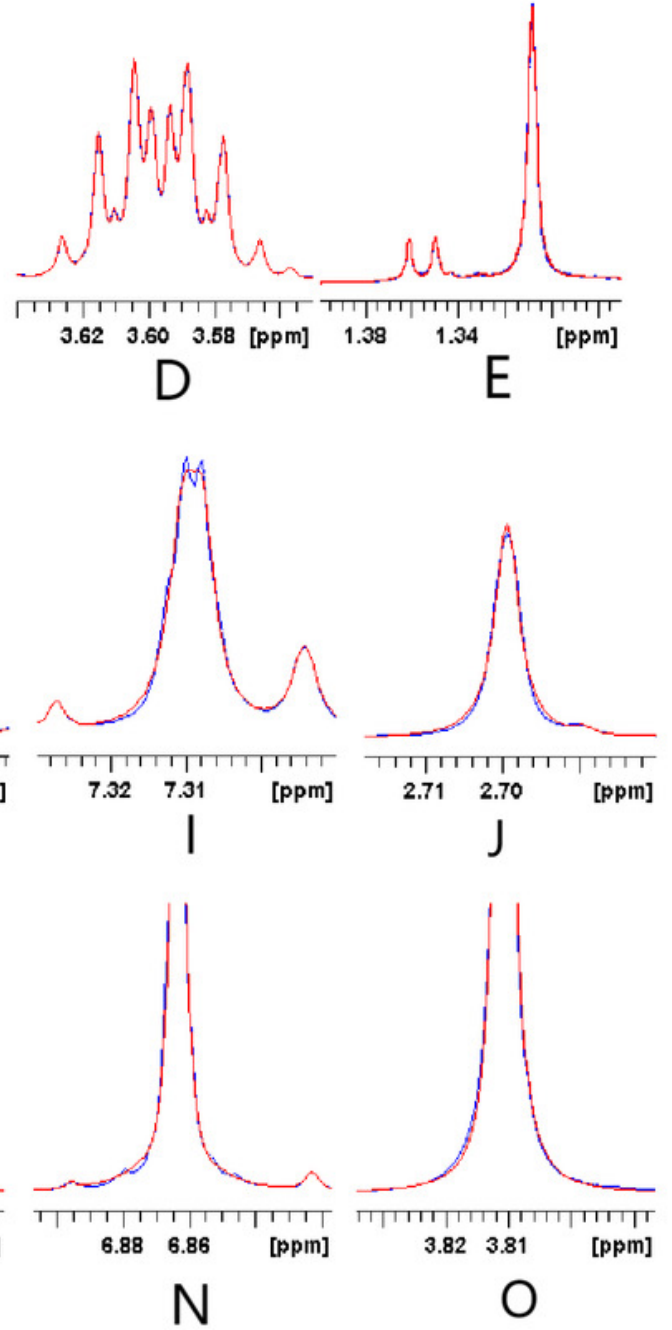
Figure 8

Figure 8 Concentrations of the three tanshinones determined by specific signals.

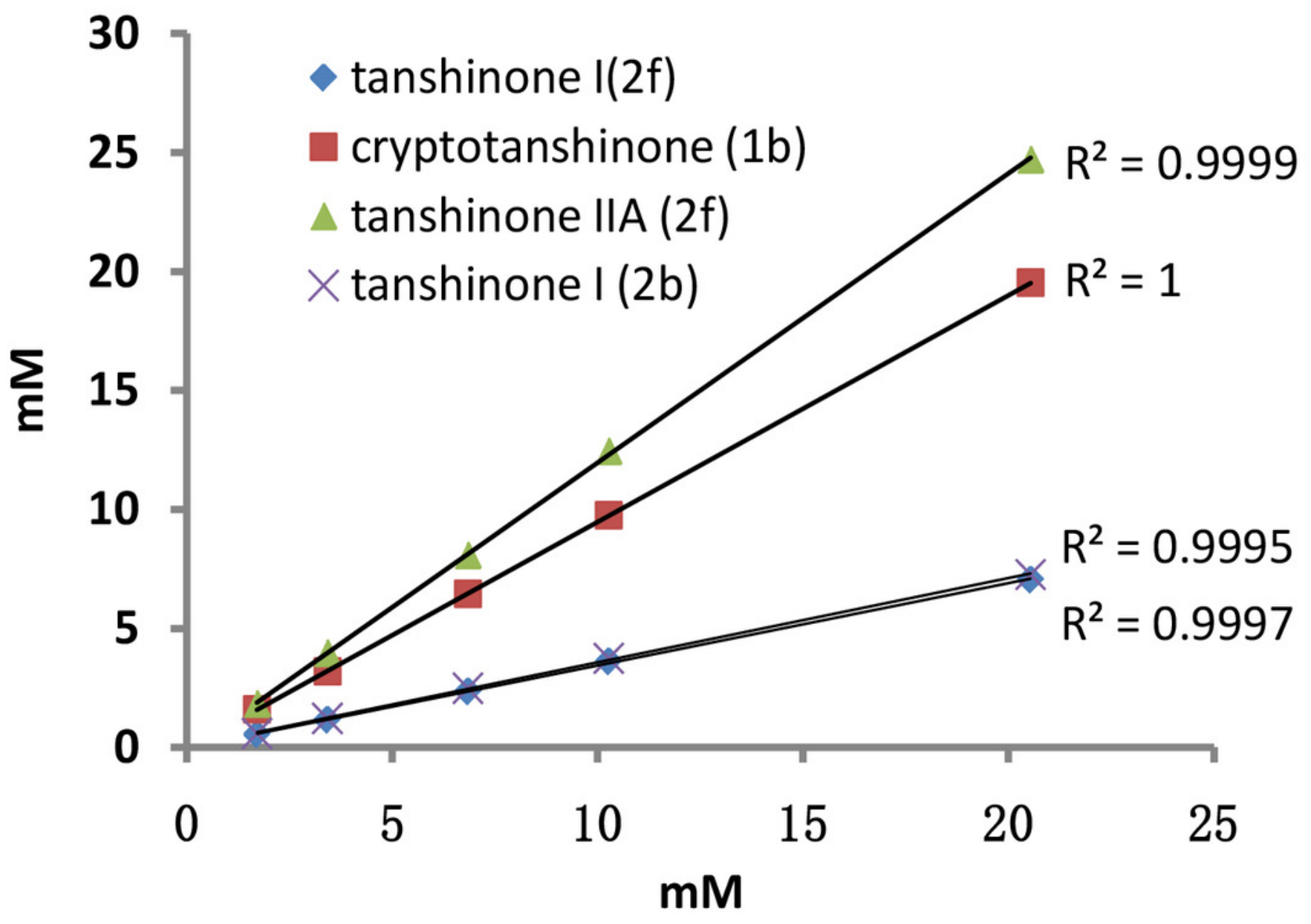


Figure 9

Deviations of the contents of the three tanshinones in the Danshen sample as determined by the MCRS contrast to individual reference standards.

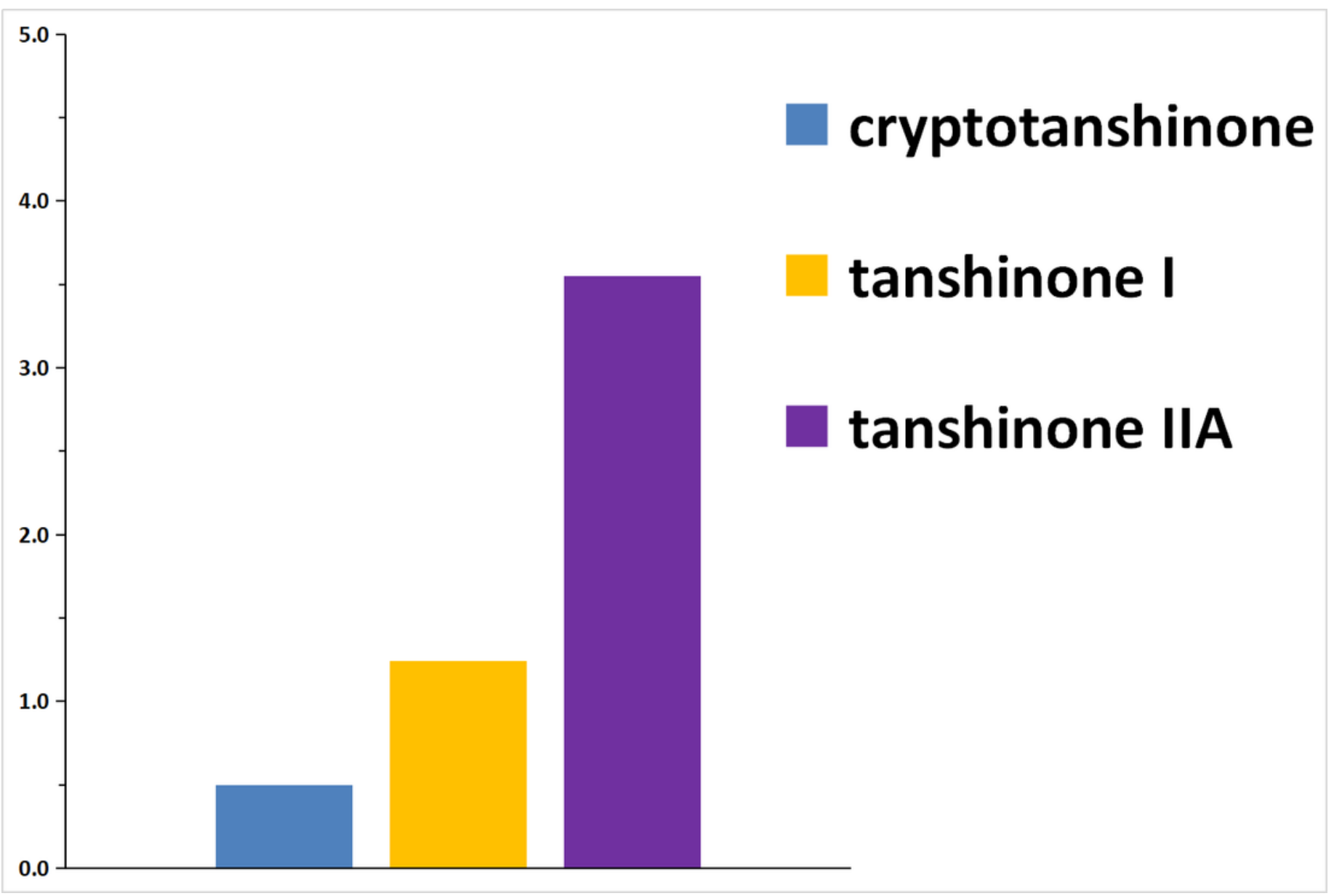

